\title{
LE GALICIEN, Une LANGUE PRISE EN ÉTAU?
}

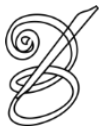 \\ Marc Pomerleau ${ }^{\mathrm{i}}$ \\ (Doutorando em Tradução - Département de Linguistique et de Traduction - \\ Université de Montréal - Montreal, Quebec, Canadá) \\ marc.pomerleau@umontreal.ca
}

Résumé : Avec plus de 240 millions de locuteurs, le portugais est sans contredit une langue de grande importance à l'échelle planétaire. Et c'est sans conteste le Portugal qui est à l'origine de sa diffusion mondiale. Toutefois, on oublie trop souvent que cette langue n'est pas exactement née dans le territoire actuel du Portugal, mais bien dans le nordouest de la péninsule ibérique, plus précisément dans la région qui constitue aujourd'hui la Galice. L'émergence du Portugal et de l'Espagne en tant que royaumes puis États-nations a fait en sorte que la langue parlée dans la frange ouest de la péninsule ibérique s'est scindée en deux, donnant naissance, du moins dans l'imaginaire collectif, à deux langues : le portugais et le galicien. Après plusieurs siècles d'évolution parallèle, un courant idéologique voudrait les voir réunies. D'une part, les tenants de cette approche, les réintégrationnistes, prônent l'épuration de la langue des castillanismes et la mise en vigueur d'une norme écrite plus proche de celle du portugais. D'autre part, les isolationnistes (ou autonomistes, selon le point de vue) prétendent que le galicien est aujourd'hui une langue à part du portugais et qu'une réintégration pourrait entraîner l'étiolement et ultimement sonner le glas de cette langue en risquant son absorption par le portugais.

Mots-clés : portugais, galicien, réintégrationnisme, langues minoritaires

Abstract: With more than 240 million speakers, Portuguese is without question a major global language. And it undoubtedly owes its worldwide breadth to Portugal's history as an empire. Yet, we tend to forget that this language was not actually born in Portugal's present-day territory, but in the northwest corner of the Iberian Peninsula, more specifically in the region known today as Galicia. As a result of the emergence of Portugal and Spain as separate Kingdoms, and later Nation States, the language spoken in the western fringe of the Iberian Peninsula began to split, giving life, at least in the collective imagination, to two languages: Portuguese and Galician. After many centuries of relatively hermetic coevolution, one school of thought would like both languages to be reunited. On the one hand, proreunion voices, called reintegrationists, advocate purging the language of Castilian influence and for the implementation of a standard written Galician closer to that of Portuguese. On the other hand, the isolationists (or autonomists, depending on one's standpoint) claim that Galician is now a different language from Portuguese and that a reintegration could weaken and ultimately sound the death knell for the language by risking its complete absorption by Portuguese.

Keywords: Portuguese, Galician, Reintegrationism, Minority Languages

Resumo: Com mais de 240 milhões de falantes, o português é, indiscutivelmente, uma língua que tem uma grande importância global. E é sem dúvida a Portugal que se deve a origem dessa difusão mundial. No entanto, muita vezes esquecemos que essa língua não nasceu exatamente no território atual de Portugal e sim, no noroeste da península ibérica, mais precisamente na região que hoje conhecemos como Galiza. O fato de Portugal e Espanha terem surgido como reinos separados e, mais tarde, como Estados-Nação, fez com que a língua falada no lado oeste da península ibérica se dividisse, dando vida, pelo menos no imaginário coletivo, a duas línguas: o português e o galego. Depois de muitos séculos de evolução paralela, uma corrente ideológica gostaria que essas duas línguas se reunissem. Por um lado, os defensores dessa abordagem, os reintegralistas, advogam a expurgação dos castelhanismos da língua e a implantação de uma norma escrita mais próxima do padrão português. Por outro, os isolacionistas (ou autonomistas, dependendo do ponto de vista) alegam que o galego é atualmente uma língua diferente do português e que uma

POMERLEAU. Le galicien, une langue prise en étau?

Belas Infiéis, v. 2, n. 2, p. 85-98, 2013. 
reintegração poderia levar a um enfraquecimento e, por fim, ao desaparecimento dessa língua, correndo o risco de ser totalmente absorvida pelo português.

Palavras-chave: português, galego, reintegracionismo, línguas minoritárias

\section{Apparition de la langue}

Le latin vulgaire qui s'est développé dans le nord-ouest de la péninsule ibérique suite à l'importation du latin par les Romains allait donner naissance à une nouvelle langue à partir $d u$ Moyen Âge. Cette langue que l'on nomme aujourd'hui galaïco-portugais est attestée depuis au moins le VIII ${ }^{\mathrm{e}}$ siècle. Les historiens de la langue s'entendent pour dire que cette langue est à la base du portugais et du galicien. En fait, jusqu'à la fin du $\mathrm{XV}^{\mathrm{e}}$ siècle au moins, le galaïcoportugais est la langue commune et de culture de cette région de la péninsule ibérique, territoire correspondant grosso modo à l'ensemble de la Galice actuelle et au nord du Portugal. Le terme utilisé pour nommer cette forme ancienne de la langue est toutefois controversé en raison de sa nature anachronique. En effet, au moment de l'apparition de la langue, le territoire où elle était parlée était nommé Gallaecia, nom hérité des Romains au Ir siècle à partir du grec Kallaikia. Le nom de ce territoire allait ensuite servir à dénommer la langue qu'on y parlait, le galicien : la dénomination gallaeco pour référer à la langue remonte au XII ${ }^{\mathrm{e}}$ siècle et celle de galego, au XIII ${ }^{\mathrm{e}}$ siècle. Le terme português [portugais], dérivé du nom de la ville de Portus Cale, est apparu plus tard, soit après l'avènement du royaume du Portugal avec le couronnement du comte Afonso Henriques, Rex Portucalensis, en 1139. Par ailleurs, ce n'est que vers l'an 1500 que le terme português allait supplanter définitivement le terme galego [galicien] au sud du fleuve Minho (LECLERC, 2013).

Cela étant dit, le fait que le terme galaïco-portugais soit anachronique n'empêche en rien son emploi, les langues anciennes étant souvent baptisées a posteriori. Les noms des langues sont d'ailleurs assujettis à nombre de contingences historiques, politiques, linguistiques et autres : le terme français est un dérivé de Francs, nom d'un peuple d'origine germanique; ce qu'on nomme espagnol est à la base du castillan et pour ce qui est du chinois, il s'agit en fait d'une extraction du mandarin standard reposant sur le hànyǔ. Bref, nous avons la fâcheuse habitude d'accoler les noms de langues à des pays. Dans les faits, il est très rare que les frontières linguistiques correspondent aux frontières politiques. Et lorsque ces frontières concordent, cela est souvent le résultat de politiques d'assimilation et d'uniformisation linguistiques à l'intérieur du territoire. 
Dans le cas de la frange ouest de la péninsule ibérique, avant que n'existent le Portugal et la Galice, et de façon plus générale l'Espagne, rien ne laissait présager que la langue commune du nord-ouest allait se scinder en deux entités distinctes - portugais et galicien - du moins d'un point de vue politique. Le changement de nom de la langue au sud du Minho, découlant essentiellement du découpage géopolitique et de la volonté du Portugal de posséder une langue qui lui était propre, allait favoriser la distanciation des deux variétés de part et d'autre de la frontière. Nous verrons plus loin en quoi il n'est pas clair, d'un point de vue purement linguistique, que nous avons effectivement affaire à deux langues distinctes. Pour ce faire, nous nous réfèrerons aux travaux de nombreux chercheurs qui se sont penchés sur la question, pour la plupart Galiciens ou Portugais, dont Oscar Diaz Fouces, Xoán M. Paredes et Fernando Venâncio.

\section{Nom de la langue}

À partir du moment où le Portugal s'est constitué en royaume, puis en État-nation, il était tout à fait normal et justifié que les dirigeants veuillent associer le nom de leur pays à la langue parlée sur l'ensemble du territoire. Une langue unique et d'apparence autochtone allait permettre d'assurer la cohésion au sein des frontières du pays et le rayonnement de sa langue. Il aurait été inconcevable pour le Portugal que sa langue porte le nom de galicien, ce terme provenant d'un territoire outre-frontières. Il ne s'agit pas ici de prétendre que le Portugal ait consciemment décidé d'appeler sa langue le portugais pour des raisons d'État, mais il est clair qu'il était important pour le Royaume, puis l'Empire d'avoir «sa » langue, et non d'avoir une langue en partage avec une autre nation. La langue allait devenir un instrument national et il fallait que son nom colle avec celui de l'Empire afin d'assurer la cohésion et l'unification (BAGNO, 2011, p. 35). D'ailleurs, c'est notamment parce qu'elle s'est fait connaitre par le biais du Portugal que la langue est connue internationalement comme le portugais, tout comme le castillan, devenu l'espagnol du fait d'avoir été diffusé par l'Espagne. Si le sort de la Galice avait été autre, la langue aurait fort bien pu prendre le nom de galicien et être diffusée internationalement sous cette dénomination.

L'apparition du royaume du Portugal et l'incorporation de la Galice dans le royaume de Castille-et-León seront donc les deux événements les plus déterminants pour le futur de la langue parlée dans la frange ouest de la Péninsule. Au-delà de la question de dénomination, c'est la langue même qui empruntera deux voies pratiquement opposées à partir de l'édification de la 
frontière politique. En effet, en quelques siècles, la langue parlée au sud du fleuve Minho allait devenir une langue officielle, codifiée et prestigieuse, la langue d'un empire s'étendant tour à tour en Amérique, en Afrique et en Asie.

Au nord du Minho, la même langue allait connaître un destin tout autre, celui d'une langue reléguée à des usages restreints, sans pouvoir et sans diffusion. En fait, du $X^{\mathrm{e}}$ siècle au $\mathrm{XIX}^{\mathrm{e}}$ siècle, le galicien traversera une période de déclin appelée les siècles obscurs, pendant lesquels la langue restera confinée à un usage oral et populaire; une langue non codifiée, sans littérature écrite propre ni traductions (LAFARGA et PEGENAUTE, 2010, p. 436). Son poids économique et politique était, à toute fin pratique, nul. Le castillan, langue du pouvoir et de l'administration en Espagne, allait peu à peu remplacer le galicien dans les plus hautes sphères de la société, soit par un processus de substitution linguistique chez les locuteurs ou par le remplacement de la noblesse autochtone par une noblesse castillane. En fait, les langues dites « régionales » du Royaume n'avaient que peu d'espace : partout en Espagne, le castillan était la langue des élites, de l'administration et du pouvoir. Et bien sûr la seule langue digne d'être écrite.

\section{Distanciation}

Un tel contexte géopolitique ne pouvait qu'accélérer le processus de distanciation entre le portugais et le galicien déjà amorcé avec la Reconquête, laquelle avait entraîné le déplacement de la langue, puis de la norme, vers le sud. En effet, le pouvoir politique se fixant pour de bon à Lisbonne au XIII ${ }^{\mathrm{e}}$ siècle, la variété de langue parlée dans la capitale est devenue synonyme de «langue portugaise », faisant des variétés parlées au nord et en Galice de simples variétés périphériques. Ainsi, certains traits linguistiques du nord jusque-là considérés «normaux » ont été évacués de la nouvelle norme (BAGNO, 2011, p. 38).

Dans la foulée de la Reconquête, qui prend fin avec la prise de Faro en 1249, le galaïcoportugais entre en contact avec les populations du sud qui parlaient d'autres langues, principalement des variétés de mozarabe, une langue romane, et probablement aussi, mais de façon restreinte, l'arabe et l'amazigh. À la même époque, l'édification de la frontière politique septentrionale entre les royaumes du Portugal et d'Espagne allait devenir une frontière linguistique difficile à surmonter. Cela ne veut pas pour autant dire que le continuum linguistique s'étendant de La Corogne à Faro sera complètement brisé. Selon Venâncio (2006), il existe encore aujourd'hui tout un continuum linguistique dans la frange ouest de la péninsule ibérique, 
de sorte qu'il est même difficile de distinguer où se termine le galicien et où commence le portugais (PAREDES, 2010).

\section{Similitudes}

La force du temps n'aura donc pas brisé ce lien, ce qui démontre en partie que le portugais et le galicien appartiennent au même système. Selon la typologie mise de l'avant par le dialectologue Joan Veny (1982), on pourrait considérer que le galicien est le dialecte constitutif de la langue, c'est-à-dire qu'il en est le point de départ, alors que le portugais en est le (un) dialecte consécutif. Venâncio (2006, p. 5) précise quant à lui que le portugais et le galicien ne peuvent être séparés en tenant compte des caractéristiques (phonologiques, lexicales, morphologiques et syntaxiques) qui permettent habituellement de distinguer deux langues. En fait, le portugais et le galicien partagent les caractéristiques qui permettent de les distinguer comme un tout en regard des autres langues romanes. D'ailleurs, plusieurs termes n'existent qu'en portugais et en galicien, c'est-à-dire sans parallèle dans les autres langues romanes, comme les verbes cheirar, esquecer et lembrar. On estime qu'environ $85 \%$ du vocabulaire des deux langues est commun (LECLERC, 2013). Selon Paredes (2010), le rythme musical et la cadence du galicien, surtout chez les locuteurs âgés dont la langue est moins marquée de traits castillans, peuvent facilement berner un lusophone au point de lui faire croire que le locuteur galicien est en fait originaire du nord du Portugal. Xoan Paredes, fils d'immigrants galiciens ayant vécu aux Baléares et en Irlande, explique d'ailleurs que lorsqu'il passait des vacances en Galice et au Portugal, il communiquait librement aux deux endroits, sans se demander s'il y avait des différences entre le galicien et le portugais.

\section{Différences}

Le grand nombre de similitudes entre le portugais et le galicien ne signifie pas pour autant qu'ils sont identiques, ce qui serait d'ailleurs impossible, la variation étant une caractéristique intrinsèque de toute langue. Il existe en effet plusieurs différences : certaines sont anciennes et relèvent de la dynamique interne de la langue alors que d'autres sont plus récentes et découlent essentiellement du contact entre le galicien et le castillan.

Comme nous l'avons vu plus tôt, dès le Moyen Âge, le portugais a été influencé par les langues parlées au sud, essentiellement le mozarabe, ce qui n'a pas été le cas pour le galicien. 
L'importance de ce substrat n'est toutefois pas majeure dans la différentiation des deux variétés. L'élément différenciateur le plus marquant se situe au niveau de l'influence du castillan sur le galicien depuis l'incorporation de la Galice au royaume de Castille puis à l'Espagne. Les castillanismes sont particulièrement nombreux dans la langue populaire parlée dans les villes, surtout chez les jeunes. Cela est notamment attribuable aux médias et aux nouvelles technologies, dont la présence en castillan est beaucoup plus forte qu'elle ne l'est en galicien. Qui plus est, les nouveaux termes liés aux sciences et technologies sont le plus souvent des emprunts au castillan, langue qui avait déjà commencé à fournir amplement de termes dans les domaines de l'économie et de la politique, entre autres. Le galicien possède également certains traits propres, notamment aux niveaux phonétique, grammatical et lexical. Sur ce dernier élément, Paredes affirme que certains termes en usage en Galice sont en fait des archaïsmes, c'est-à-dire des termes aujourd'hui désuets dans le portugais standard, alors que d'autres sont des régionalismes qu'on retrouve parfois également dans certains parlers du nord du Portugal. C'est notamment pour cette raison que le galicien est parfois considéré comme étant plus conservateur que le portugais (PAREDES, 2010). Du côté de la phonétique, la marque laissée par le castillan est telle que pour de nombreux Portugais, à l'oral le galicien ressemble à du portugais parlé avec un accent espagnol. Cette observation est toutefois réductrice et il serait démesuré d'avancer que toutes les spécificités phonétiques du galicien sont attribuables à l'influence du castillan.

Selon les partisans de l'approche réintégrationniste, ces quelques différences ne justifient aucunement les prétentions de ceux qui voient le galicien comme une langue distincte du portugais. En effet, et comme le précise Venâncio (2006, p. 7), les différences entre le galicien et le portugais ne sont pas plus importantes que celles qu'on relève par exemple entre certaines variétés du portugais parlées au Portugal et au Brésil, ou ailleurs dans le monde lusophone. Et pourtant, personne ne prétend que les Brésiliens ne parlent pas portugais. Júlio César Barreto Rocha, professeur à l'Universidade Federal de Rondônia, va jusqu'à affirmer qu'on pourrait avancer que lorsque l'on parle du portugais, on pourrait tout aussi bien parler de la variété portugaise du galicien. Et pour ce qui est du Brésil, on pourrait dire qu'on y parle un dialecte du galicien (BARRETO ROCHA, 1997).

Au-delà des différences phonétiques, grammaticales et lexicales déjà mentionnées, l'élément différenciateur le plus marquant entre le portugais et le galicien se situe au niveau de la graphie. Alors que le portugais a été codifié dès le $\mathrm{XVI}^{\mathrm{e}}$ siècle, le galicien, principalement en 
raison de son caractère non officiel et de la nette domination du castillan à l'écrit en Galice, n'a été décrit par aucune grammaire avant le XIX ${ }^{\mathrm{e}}$ siècle et n'a pas été officiellement codifié avant la fin du $\mathrm{XX}^{\mathrm{e}}$ siècle. Même pendant la renaissance de la littérature galicienne au XIX ${ }^{\mathrm{e}}$ siècle, faute de norme écrite, les auteurs galiciens écrivaient leur langue à l'aide de la graphie castillane, la seule qu'ils connaissaient, la seule enseignée à l'école.

\section{Norme RAG}

Cette situation, renforcée par l'interdiction de l'enseignement du galicien durant le Franquisme, a fait en sorte que la plupart des Galiciens étaient jusqu'à récemment illettrés dans leur propre langue. Ce n'est qu'avec le retour de la démocratie au début des années 1980 que le galicien a finalement été normalisé. Toutefois, la norme officielle développée par l'Académie royale galicienne (RAG) et l'Institut de la langue galicienne (ILG) ne fait pas consensus au sein de la population, du moins au sein de certains secteurs, essentiellement nationalistes. D'abord fixée en 1982 puis mise à jour en 1995 et 2003, cette norme a été en partie établie à partir de la graphie castillane. Ce choix a causé des remous au sein des secteurs réintégrationnistes, ces derniers prônant une graphie plus proche de celle du portugais, tel que proposé par l'Association galicienne de la langue (AGAL). Selon Paredes (2010), la norme officielle « à l'espagnole » a été choisie afin d'être plus facilement assimilable pour les Galiciens, jusque-là essentiellement scolarisés en castillan et beaucoup plus habitués à lire le castillan que le portugais, et ce, malgré la similitude entre cette langue - ou variété de langue - et la leur. À ce jour, la norme officielle est de loin la plus utilisée : elle est celle de l'administration, de la grande majorité des médias écrits et de l'enseignement, ce dernier élément faisant en sorte que toutes les personnes scolarisées en Galice utilisent systématiquement la norme officielle, un avantage d'envergure pour les tenants de cette approche. Ainsi, on préconise l'utilisation du 'll' plutôt que du 'lh' et du 'ñ' plutôt que du 'nh', de même que du “s" simple là où le portugais utilise souvent le 'ss', et ainsi de suite. On accepte donc les conventions d'écriture de la langue dominante au détriment de l'écriture historico-étymologique, ce que bon nombre de réintégrationnistes appellent la «vraie graphie patrimoniale »(CASTRO et al., 2007, p. 16). En raison de cette norme basée sur la graphie castillane, beaucoup de lusophones, lorsqu'on leur présente un texte en galicien, ont l'étrange sensation d'avoir devant les yeux un texte écrit en portugais, mais dans lequel on aurait 
inséré de drôles de lettres. Pour d'autres, l'orthographe normative du galicien leur donne la sensation d'être en présence d'une langue intermédiaire, mi-espagnole et mi-portugaise.

\section{Norme AGAL}

La voie réintégrationniste prônée par l'Association galicienne de la langue vise un retour aux racines par la récupération de l'écriture historico-étymologique. Cette approche vise notamment à épurer le galicien de ses éléments castillans par de nouveaux efforts de codification. Le réintégrationnisme ne prône pas un alignement complet sur l'orthographe portugaise contemporaine, mais quelques modifications qui permettraient un rapprochement, tout en conservant les différences pertinentes : utilisation du 'nh', très fréquent en portugais à la place du 'ñ', lettre phare du castillan pratiquement devenue marque de commerce de l'Espagne ${ }^{\mathrm{iv}}$; remplacement du 'ción' par le 'çom', graphie reprise du galicien médiéval qui se distingue du ‘ção' portugais pour des questions phonétiques. Le tableau suivant présente quelques-unes des différences actuelles :

\section{Tableau comparatif}

\begin{tabular}{|l|l|l|l|}
\hline \multicolumn{1}{|c|}{ Portugais } & \multicolumn{1}{|c|}{$\begin{array}{c}\text { Galicien } \\
\text { réintégrationisme }\end{array}$} & Galicien normatif & \multicolumn{1}{c|}{ Espagnol } \\
\hline $\begin{array}{l}\text { Decreto de } \\
\text { Normativização da } \\
\text { Língua Galega }\end{array}$ & $\begin{array}{l}\text { Decreto de } \\
\text { Normativizaçom da } \\
\text { Língua Galega }\end{array}$ & $\begin{array}{l}\text { Decreto de } \\
\text { Normativización da } \\
\text { Lingua Galega }\end{array}$ & $\begin{array}{l}\text { Necreto de } \\
\text { Normativización de } \\
\text { la Lengua Gallega }\end{array}$ \\
\hline Bem-vindo & Bem-vido & Benvido & Bienvenido \\
\hline Amo-te & Amo-te & Ámote & Te amo \\
\hline
\end{tabular}

\section{Réintégrationnisme}

Le réintégrationnisme vise également à redorer l'image que les Galiciens se font de leur langue en participant à la déconstruction du mythe selon lequel le galicien est une langue régionale sans aucune utilité à l'échelle internationale. Les réintégrationnistes croient en effet qu'en adoptant une norme proche de celle du portugais, les Galiciens pourraient avoir la sensation de parler une langue qui a droit de cité, de calibre international et en partage avec plus 
de 240 millions de locuteurs en Amérique, en Afrique et en Asie. De plus, en adoptant une norme «à la portugaise » et en empruntant à cette langue au besoin, le galicien aurait moins tendance à se castillaniser, une tare aux yeux des réintégrationnistes. Ces derniers se défendent d'ailleurs d'avoir des visées essentiellement politiques, insistant sur le fait que leur vision de la langue est d'abord scientifique et fondée sur l'essence de la langue galicienne. Pour eux, ce sont plutôt les isolationnistes qui ont des visées politiques, voulant reléguer la Galice et sa langue à des rôles de

seconde zone. À ce chapitre, n'oublions pas que la langue - toute langue - est un outil maniable, pour ne pas dire manipulable, utilisé entre autres à des fins politiques. En ce sens, il serait surprenant que l'un ou l'autre de ces deux pôles diamétralement opposés n'ait aucune visée politique, que ce soit consciemment ou non.

D'aucuns pensent que le réintégrationnisme est un phénomène récent, mais dans les faits dès 1726 le père Feijoo affirmait l'unité linguistique galaïco-portugaise (CASTRO et al., 2007). À la fin du $\mathrm{XIX}^{\mathrm{e}}$ siècle et au début du $\mathrm{XX}^{\mathrm{e}}$, des activistes galiciens réintégrationnistes exilés faisaient la promotion de cette voie depuis Buenos Aires et La Havane, notamment.

\section{La lusophonie}

Pour les réintégrationnistes, créer de nouveaux liens avec la lusophonie constitue la voie à privilégier afin de faire du galicien une langue internationale. Pour ce faire, les tenants de cette approche souhaitent que la Galice devienne l'un des membres officiels de la Communauté des pays de langue portugaise, qui compte à ce jour huit pays membres ${ }^{\mathrm{v}}$. Notons que par le passé le gouvernement autonome de la Galice a déjà amorcé certaines démarches afin d'adhérer à l'organisation, que ce soit comme gouvernement observateur ou autre, mais que rien de concret n'a été proposé pour l'instant. Dans les faits, et selon Xosé-Henrique Costas, la Galice occupe déjà une position de choix au sein de la lusophonie. Dans son livre-choc 55 mentiras sobre a lingua galega ${ }^{v i}$, il déboulonne le mythe voulant que le galicien n'ait pas de portée internationale en démontrant que cette langue facilite énormément les relations d'affaires avec le Portugal et le Brésil : les Galiciens sont de loin préférés aux autres Espagnols par les entreprises portugaises et brésiliennes parce qu'ils « connaissent déjà la langue »(COSTAS, 2010, p. 72).

Parmi les stratégies que les réintégrationnistes voudraient mettre de l'avant, notons les échanges universitaires et culturels avec le Portugal, la mise en place de festivals de cinéma, de bourses d'études, de prix littéraires conjoints, etc. En somme, on voudrait que le pays se tourne 
davantage vers le sud que vers l'est, et que les Galiciens aient plus facilement accès aux productions culturelles portugaises en particulier et lusophones en général, comme les livres, les émissions de radio et de télévision (l'offre de chaînes de télévision portugaises en Galice est pratiquement inexistante). On propose également que le système scolaire galicien offre des cours de portugais à tous les élèves, facilitant l'apprentissage en un court laps de temps par une majorité de Galiciens du portugais standard et sa cohabitation avec le galicien. Ils auraient ainsi encore plus facilement accès aux productions culturelles lusophones. Après tout, pourquoi les Galiciens devraient-ils se priver des ouvrages écrits dans la langue de Camões, dont la famille même était d'origine galicienne? ${ }^{\mathrm{vii}}$

Du côté des isolationnistes, - qui préfèrent s'auto-qualifier d'autonomistes ou de différentialistes - on prétend qu'une intégration de la Galice dans l'aire lusophone pourrait entraîner à long terme une absorption du galicien par le portugais, ce à quoi les réintégrationnistes rétorquent que c'est exactement ce que le castillan est en train de faire au galicien. Selon Diaz Fouces, (2003), les isolationnistes refusent d'explorer la voie de la lusophonie en affirmant que les Galiciens disposent déjà d'un accès privilégié au monde via l'espagnol.

\section{L'Espagne}

Depuis des siècles, l'administration espagnole fait des pieds et des mains pour que les principales langues autochtones non castillanes parlées sur son territoire, soit le galicien, le catalan et le basque, n'entrent pas en concurrence avec le castillan, langue de l'État et, selon l'article 3 de la Constitution espagnole, seule langue que les citoyens ont le devoir de connaître. Ces trois langues, co-officielles avec le castillan dans les régions où elles sont parlées, ne sont considérées que comme des langues régionales ou «autonomiques », c'est-à-dire propres à certaines Communautés autonomes au sein de l'Espagne, non aptes à servir les intérêts de l'État outre-frontières. S'il s'avérait que l'une de ces langues, le galicien dans le cas qui nous intéresse, soit considérée comme une langue internationale de par sa proximité avec le portugais, cela viendrait troubler la conception actuelle selon laquelle seul le castillan puisse être une langue de grande diffusion et de portée internationale. Alors que l'Espagne pourrait tirer profit de cette langue qui lui ouvrirait la porte de la lusophonie, elle préfère confier à la langue de l'État la tâche unique d'internationalisation. Pour les nationalistes espagnols, une reconnaissance du galicien en 
tant que langue internationale par le biais du portugais pourrait même provoquer un éveil de la conscience nationale galicienne et menacer l'unité de l'Espagne. Bref, même si d'un point de vue strictement linguistique il est évident que le portugais et le galicien sont très proches, les garder politiquement séparés permet en outre d'éviter d'éventuels conflits internes en Espagne.

\section{Le Portugal}

$\mathrm{Au}$ Portugal, on considère généralement que l'histoire de la nation portugaise commence avec l'instauration du Royaume, ce qui a pour conséquence de sous-estimer l'apport de la Galice dans l'histoire du pays, et surtout dans la formation de sa langue nationale. Dans l'imaginaire collectif, les frontières du pays correspondent parfaitement à celles de la langue. C'est cette vision du Portugal que Venâncio (2006) caractérise de rupture : les Portugais, davantage que les Espagnols, qui sont à tout le moins conscients de la diversité présente au sein des frontières de leur État, voient leur pays comme un tout homogène. Il leur est donc difficile de se représenter l'Espagne comme un conglomérat de peuples aux langues diverses, ce qui explique en partie leur méconnaissance du lien de parenté entre leur langue et leur culture avec celles de la Galice. En fait, la question galicienne préoccupe peu le Portugal, qui n'a pas de politique officielle sur la Galice ou le galicien. Les réintégrationnistes croient que la réintégration de la langue galicienne dans son aire d'origine pourrait favoriser un nouvel enthousiasme chez les Portugais, qui se sentiraient davantage concernés par ce qui se passe en Galice. Il ne faut toutefois pas oublier que le Portugal est dans une situation délicate, ne voulant s'aliéner ni la Galice ni l'Espagne : c'est ce que Venâncio (2006) appelle la « double loyauté ».

\section{Conclusion}

En conclusion, la langue galicienne semble prise dans un étau entre deux pôles opposés (réintégrationnistes et isolationnistes) et entre deux géants (l'espagnol et le portugais). Le Galicien est-il arrivé à la croisée des chemins? Quelle voie la langue prendra-t-elle? Celle de la norme officielle semble avoir une bonne longueur d'avance, ce qui ne signifie pas qu'un rapprochement avec le portugais est impossible. Il existe entre le réintégrationnisme et l'isolationnisme un éventail de possibilités. Le galicien pourrait fort bien continuer à faire cavalier seul, que ce soit par le statu quo, ce qui sous-entend l'acceptation de sa castillanisation ancienne, actuelle et à venir, principal élément qui le différencie du portugais, mais élément qui 
pourrait constituer une menace à sa pérennité à long terme. Les autorités pourraient également mettre en œuvre de nouvelles politiques linguistiques favorables à sa rétention, à sa transmission et à sa diffusion en tant que langue propre de la Communauté autonome. On pourrait aussi recourir davantage au portugais pour contrebalancer la pression du castillan, ce qui ne se fera pas sans volonté politique en ce sens. Qu'à l'avenir la langue penche davantage vers le portugais ou non, les locuteurs du galicien ont déjà l'avantage de pouvoir jouer sur deux fronts, soit ceux de l'hispanophonie et de la lusophonie.

\section{Bibliographie}

ASSOCIAÇOM GALEGA DA LÍNGUA. O que é o PGL? Portal Galego da Língua. Santiago de Compostela. Disponible à http://www.pglingua.org/acerca-do-pgl. Consulté le 4 juin 2013.

BAGNO, Marcos. O português não procede do latim: uma proposta de classificação das línguas derivadas do galego. Grial: revista galega de cultura, n. 191, p. 34-39, 2011.

BARRETO ROCHA, Júlio César. O Brasil fala a língua galega. Çopyright, A Corunha, n. 44, julho 1997.

BOYER, Henri. Le nationalisme linguistique : une option interventionniste face aux conceptions libérales du marché de langues. Noves SL. Revista de Sociolingüística, Barcelone, automnehiver 2006.

CASTRO, Mauricio; RODRIGUES FAGIM, Valentim et al. Reintegracionismo lingüístico: identidade e futuro para o galego. Voz Própria, n. 19, p. 15-32, 2007.

COSTAS, Xosé-Henrique (Ed.). 55 mentiras sobre a lingua galega. Santiago de Compostela: Laiovento, 2010.

DIAZ FOUCES, Oscar. Políticas de traducción en Cataluña y Galicia. Communication présentée au $5^{e}$ colloque sur la traduction, la terminologie et l'interprétation à Cuba et au Canada. La Havane, 8 décembre 2004.

DIAZ FOUCES, Oscar. La codificació del gallec o el pèndol que no s'atura. Quaderns d'Italià, n. 8/9, 2003/2004.

DIAZ FOUCES, Oscar. Apontamentos sobre a socializaçom do Reintegracionismo. Agália, n. 67-68 (B), p. 9-34, 2001. 
FAKTOROVÁ, Simona. O galego ontem e hoje em comparação com o português. Mémoire (Maîtrise en Langues et littératures romanes) - Université Masaryk, Brno, République tchèque, 2011.

HERRERO-VALEIRO, Mário J. The discourse of language in Galiza: Normalisation, diglossia, and conflict. Estudios de Sociolinguiística, Vigo, n. 3 (2) 2002 \& 4(1) 2003, p. 289-320, 2003.

GEMIE, Sharif. A concise History of Galicia. Cardiff: University of Wales Press, 2006.

LAFARGA, Francisco; PEGENAUTE, Luis (Ed.) Diccionario histórico de la traducción en España. Madrid: Editorial Gredos, 2010.

LANE GREENE, Robert. You Are What You Speak: Grammar Grouches, Language Laws, and the Politics of Identity. New York: Delacorte Press, 2011.

LECLERC, Jacques. Galice. L'aménagement linguistique dans le monde, Québec, TLFQ, Université Laval, 4 mars 2013. Disponible à

http://www.axl.cefan.ulaval.ca/europe/espagnegalice.htm. Consulté le 4 juin 2013.

MILlÁN-VARELA, Carmen. Translation, Normalisation and Identity in Galicia(n). Target, n. 12:2, p. 267-282, 2000.

NOGUEIRA, Camilo. O Galego-Português-Brasileiro e a Política Linguística na Galiza. Agália, n. 62. p. 112-118, 2000.

PAREDES, Xoán M. A dicotomia galego/português é a mesma que a castelhano/espanhol (entrevue). Portal Galego da Língua. Santiago de Compostela: Associaçom Galega da Língua, 2010. Disponible à

http://www.pglingua.org/index.php?option=com_content\&view=article\&id=2029:xoan-paredesgeografo-e-musico-baterista\&catid=22:agal-hoje\&Itemid=61. Consulté le 12 juin 2013.

PAREDES, Xoán M. A língua portuguesa da Galiza. Santiago de Compostela: Associaçom Galega da Língua, 2006. Disponible à http://www.xoan.net/recursos/Pt_da_Gz.pdf. Consulté le 12 juin 2013.

VENÂNCIO, Fernando. Mana Galiza. Expresso, n. 1831, 1er décembre 2007.

VENÂNCIO, Fernando. "I see my language everywhere": On linguistic relationship between Galicia and Portugal. Communication présentée à Cork, Irlande, le 21 mars 2006. Disponible à http://www.agal-

gz.org/modules.php?name=IndyNews\&MediaOp=get\&idMediaAss $=$ s2790\&MediaName $=$ venan cio_cork_03_2006.pdf\&MediaNum=1. Consulté le 12 juin 2013.

VENÂNCIO, Fernando. A Galiza, A Espanha e nós. Visão, 20 octobre 2005. 
VENY i CLAR, Joan. Els parlars catalans. Síntesi de dialectologia. Palma de Majorque: Moll, 1982.

Index Translationum. Disponible à <http://portal.unesco.org/culture/fr/ev.phpURL_ID=7810\&URL_DO=DO_TOPIC\&URL_SECTION=201.html. Consulté le 27 mai 2013.

\footnotetext{
${ }^{\text {i }}$ Marc Pomerleau - Traducteur agréé. Disponível em: http://mpom.ca/formation/

ii «O assunto é que eu ia à Galiza ou a Portugal de férias e comunicava livremente tanto num sítio como noutro. Não pensava mais no tema e era frequente que os meus amigos não galegos me pedissem para traduzir isto ou aquilo do português porque eu, como galego, "sabia português". » (Paredes, 2010)

iii En préparation de cet article, j'ai consulté plusieurs articles en portugais et en galicien. La lecture des articles en galicien n'a posé aucun problème, même si je n'ai jamais étudié cette langue. En fait, en cours de lecture, je me demandais parfois si l'article sous mes yeux était en portugais ou en galicien, tellement les deux « langues »se ressemblent.

iv Le 'ñ' a notamment été intégré dans le logo de l'Institut Cervantes. Voir www.cervantes.es.

${ }^{v}$ Pays membres (2013) : Angola, Brésil, Cap-Vert, Guinée-Bissau, Mozambique, Portugal, Sao Tomé-et-Principe, Timor oriental.

${ }^{\text {vi }}$ Traduction libre : 55 mensonges sur la langue galicienne.

98 vii La famille de Luís de Camões, figure emblématique de la littérature de langue portugaise et auteur des Lusiades, était originaire du cap Finisterre en Galice. L'un de ses ancêtres était le troubadour galicien Vasco Pires de Camões.
} 